\title{
Low Latency LDGM Code for Multimedia-Packet Stream in Bursty Packet Loss Networks
}

\author{
Filippo Casu, Julián Cabrera, Fernando Jaureguizar, and Narciso García
}

\begin{abstract}
In this paper we present a FEC scheme based on simple LDGM codes to protect packetized multimedia streams. We demonstrate that simple LDGM codes working with a limited number of packets (small values of $k$ ) obtain recovery capabilities, against bursty packet losses, that are similar to those of other more complex FEC-based schemes designed for this type of channels.
\end{abstract}

\section{INTRODUCTION}

In the last years multimedia communication are moving to IP networks, since they are more efficient than circuit switching channels. Nevertheless, IP networks have some disadvantages depending on the packets delivery and the delays control [1].

In this paper we focus on the packet delivery problem (erasure channels). In order to avoid it, two protection techniques exist, ARQ (Automatic Repeat reQuest) based schemes and FEC (Forward Error Correction) based schemes. FEC techniques consist in generating redundancy that can be used to recover lost information. ARQ techniques are based on the retransmission of lost information.

FEC-based schemes are usually preferred to ARQ schemes in time-sensitive communication, since no extra delay is added due to the retransmission of lost information [2].

Different examples of FEC codes employed in packetized multimedia streams are: XOR-based (eXcluive OR) codes, simple and interleaved, Low-Density Parity-Check (LDPC) codes, Reed-Solomon codes, etc.

In this paper we focus on a family of LDPC codes, the Low-Density Generator-Matrix (LDGM) codes, since they require a low computational cost at both, the decoding and encoding stages. In fact, LDGM codes need encoding/decoding times lower than the classical LDPC codes. This characteristic can be very useful in multicast transmission scenarios with different types of receiver that have a limited computational capability.

In this work we demonstrate for an ADSL simulated channel, that the simple case of LDGM code working with small groups of packets is able to obtain recovery capabilities similar to more complex FEC codes designed for bursty packet losses: a FEC scheme based on interleaving matrix, proposed by the Pro-MPEG forum in its COP \#3 [3], and a FEC scheme based on Reed-Solomon codes.

\section{LOW LATENCY LDGM CODES}

\section{A. $L D P C$ codes}

LDPC codes belong to the class of linear block codes defined by parameters $k$, and $n$, which corresponds to the number of bits of an information vector and code vector respectively. Thus, the number of redundancy bits is $n-k$.

An LPDC code is defined by its parity matrix $H$ of dimensions $n-k$ and $n$, that holds equation (1):

$$
\mathbf{c} H^{T}=\mathbf{0}
$$

where $\mathbf{c}$ is a code vector. The parity-check is so named because it provides $n-k$ parity check equations on a received vector [4].

A LDPC codes is a linear block code for which the paritycheck matrix $H$ has a low density of 1 's.

From $H$ we are able to construct the so called generator matrix $G$ that allows the generation of the redundancy from the information vector following equation (2):

$$
\mathbf{c}=\mathbf{u} G
$$

where $\mathbf{c}$ is a code vector, and $\mathbf{u}$ an information vector.

Although these codes do not belong to the family of MDS (Maximum Separable Code), that means they do not offer the best recovery capability for a block code, they require a very low computational complexity. This characteristic depends on two reasons: the using of XOR operation to generate the redundancy, and the low density of the generator matrix that defines a low number of operations.

Moreover, LDPC codes are defined as large-block codes, reaching the best recovery capabilities for very big values of $k$ (about $1000[5]$ ).

\section{B. $L D G M$ codes}

A simplified version of LDPC codes is represented by LDGM codes, for which the parity-check matrix $H$ corresponds also to the generator matrix $G$ [5].

The most important distinction between these two types of codes is that the encoding/decoding operations are faster with LDGM than with LDPC. Nevertheless LDPC has a major advantage from the error correction capability point of view.

If we consider a multicast transmission with different receivers that have a limited computational capability, it is necessary to employ FEC codes whose decoding time is very low, as is the case of LDGM codes. Moreover, LDGM codes encoders/decoders are easy to implement and different implementations are available.

Another important aspect is the latency generated by big values of $k$ : it becomes a problem in case of time sensitive services, such as videoconference, so it is very useful 
employing low values of this parameter.

\section{LDGM CODES IN LOW-LATENCY MEMORY CHANNELS}

LDGM codes are usually used in memoryless channels with high values of $k$ in order to effectively protect from packet losses. Nevertheless, in this paper we address the use of LDGM codes for channels where losses are produced in bursts and where it is necessary to keep a low latency.

Our protection scheme consists in dividing the total packets needed to stream a given multimedia content in groups of $k$ data-packets, and generate, according to $G, n-k$ FEC-packets for each data-group of packets. In case of lost packets in a group, the FEC-packets are used to recovery the lost packets at the receiver.

We chose low values of $k$ in order to allow the use of these codes for time sensitive services: this choice depends on the rate of the content and the statistical characteristics of the transmission channel as PER (Packet Error Rate) and $L_{m}$ (average burst length).

In that sense [6] proposes as typical value of PER for an ADSL channel about $1 \%$ and average burst length, $L_{m}$, equal to 10 . It employs an interleaving protection scheme which $k$ is 80 and is able to recovery burst of 20 packets.

We have based our analysis over these values in order to test and compare our proposal scheme for three different averages burst length $(15,20$ and 25$)$, since the length of a burst in number of packets, although the time is fixed, on the rate of the transmission.

Moreover, we compare LDGM to a FEC-scheme proposed in [7] that is based on Reed-Solomon codes.

\section{SIMULATION RESULTS}

We have carried out different experiments for three different codes: XOR-Interleaving Matrix, Reed-Solomon codes based scheme, and a simple LDGM code.

Fig. 1 shows the ratio of recovered packets with respect to the lost packets. As we can observe, the Reed-Solomon codes based scheme obtains the best recovery results, however the computational cost associated to this codes is appreciably higher than the XOR-based codes.

Moreover, if we consider only XOR-based codes, LDGM outperforms the XOR-interleaving scheme as the average burst length increase.

\section{Percentage of recovered packets}

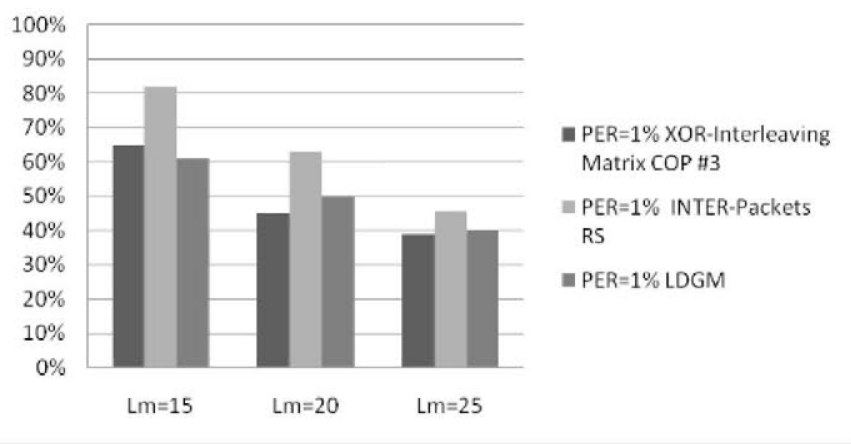

Figure 1. Percentage of recovered packets obtained by three different FEC scheme: Interleaving Matrix, Inter-packet Reed-Solomon Symbol approach, simple LDGM code.

\section{CONCLUSIONS AND FUTURE WORKS}

In this paper we have introduced the use of low latency simple LDGM codes in bursty packet loss networks. We have tested and compared a simple LDGM with two FEC schemes, designed for bursty packet loss networks, and used to protect a packetized stream in an ADSL simulated environment. We have demonstrated that a simple LDGM can reach good performances for low values of $k$ in bursty packet loss networks. Moreover, the easy implementation of this codes, covert them as a very appealing solution for real communication environment (ADSL channels, Wi-Fi channels, etc.).

Next steps will address the design of LDGM codes whose generator matrix $G$ is created considering the statistical characteristics of the channel.

\section{REFERENCES}

[1] K. R. Rao, Z. S. Bojkovic, and D. A. Milovanovic, Multimedia Communication Systems: Techniques, Standards, and Networks. Upper Saddle River, NJ, USA: Prentice Hall PTR, 2002

[2] C. Lamoriniere, A. Nafaa, and L. Murphy, "Dynamic switching between adaptive fec protocols for reliable multi-source streaming," in Global Telecommunications Conference, 2009. GLOBECOM 2009. IEEE, 30 2009.

[3] Pro-MPEG Forum, "Transmission of Professional MPEG-2 transport streams over IP networks", Code of Practice \#3 release 2, July 2004.

[4] W. E. Ryan, "An Introduction to LDPC Codes," CRC Handbook for Coding and Signal Processing for Recording Systems, Ed., B. Vasic and E. Kurtas, CRC Press, 2004.

[5] V. Roca, Z. Khallouf, J. Laboure, "Design and Evaluation of an LDGM Large Block FEC Codec," In fifth International Workshop on Networked Group Communication (NGC'03), Munich, Germany, September 2003.

[6] C. Díaz, J. Cabrera, F. Jaureguizar, and N. García, "A Video-Aware FEC-Based Unequal Loss Protection System for Video Streaming over RTP," IEEE Transaction on Consumer Electronics, vol. 57, no2, pp. 523-531, July 2011.

[7] F. Casu, J. Cabrera, F. Jaureguizar, and N. García, "Inter-Packet Symbol Approach To Reed-Solomon FEC Codes For RTP-Multimedia Stream Protection," MediaWiN, 2011. 